\title{
Reconstruction of large segmental bone defects in rabbit using the Masquelet technique with a-calcium sulfate hemihydrate
}

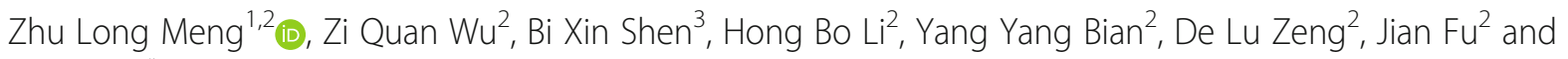
Lei Peng ${ }^{2^{*}}$

\begin{abstract}
Background: Large segmental bone defects can be repaired using the Masquelet technique in conjunction with autologous cancellous bone (ACB). However, ACB harvesting is severely restricted. a-calcium sulfate hemihydrate (a$\mathrm{CSH}$ ) is an outstanding bone substitute due to its easy availability, excellent biocompatibility, biodegradability, and osteoconductivity. However, the resorption rate of a-CSH is too fast to match the rate of new bone formation. The objective of this study was to investigate the bone repair capacity of the Masquelet technique in conjunction with isolated a-CSH or an a-CSH/ACB mix in a rabbit critical-sized defect model.

Methods: The rabbits $(n=28)$ were randomized into four groups: sham, isolated a-CSH, a-CSH/ACB mix, and isolated ACB group. A 15-mm critical-sized defect was established in the left radius, followed by filling with polymethyl methacrylate spacer. Six weeks after the first operation, the spacers were removed and the membranous tubes were grafted with isolated a-CSH, isolated ACB, a-CSH/ACB mix, or none. Twelve weeks later, the outcomes were evaluated by manual assessment, radiography, and spiral-CT. The histopathological and morphological changes were examined by H\&E staining. The levels of alkaline phosphatase and osteocalcin were analyzed by immunohistochemistry and immunofluorescence staining.
\end{abstract}

Results: Our results suggest that the bone repair capacity of the a-CSH/ACB mix group was similar to the isolated ACB group, while the isolated a-CSH group was significantly decreased compared to the isolated ACB group.

Conclusion: These results highlighted a promising strategy in the healing of large segmental bone defect with the Masquelet technique in conjunction with an a-CSH/ACB mix $(1: 1, w / w)$ as they possessed the combined effects of sufficient supply and low resorption.

Keywords: Bone defect, The Masquelet technique, a-calcium sulfate hemihydrate

\section{Background}

The treatment of large segmental bone defects caused by acute high-energy trauma, tumor destruction or resection after infection remains challenging for most orthopedic and trauma surgeons [1]. The approaches currently used to treat large segmental bone defects including autologous bone graft, vascularized free bone

\footnotetext{
*Correspondence: xiaobo197518@163.com

${ }^{2}$ Department of Trauma Center, The First Affiliated Hospital of Hainan Medical University, Haikou, China

Full list of author information is available at the end of the article
}

transfer, and the Ilizarov intercalary bone method [2]. Autologous bone graft remains the gold standard treatment for bone defects less than $5 \mathrm{~cm}$ because of their osteogenicity, osteoinductivity, and osteoconductivity [3-5]. Vascularized free bone transfer [6-8] and the Ilizarov intercalary bone method $[9,10]$ are used most commonly to treat large segmental bone defects more than $5 \mathrm{~cm}$. However, vascularized free bone transfer is microsurgically demanding. In addition, there are associated with donor site morbidity and a high failure rate [11]. The Ilizarov intercalary bone method is technically 



Fig. 1 Intraoperative photographs of the Masquelet technique. a The PMMA spacer molded in vitro. b Creation of a critical-sized defect. c Insertion of the PMMA spacer (yellow arrow) into the defect. $\mathbf{d}$ Formation of the induced membrane (red arrow). e Removal of the PMMA spacer and formation of a cavity. $\mathbf{f}$ Placement of an autologous cancellous bone graft (green arrow) into the cavity

demanding, time-consuming, and associates with a high complication rate [12].

The Masquelet technique, an emerging alternative approach first described by Alain Masquelet [13, 14], consists of a two-stage procedure that allows

Table 1. Lane-Sandhu radiographic scoring standard

\begin{tabular}{lll}
\hline Category & Standard & Scores \\
\hline Callus & no callus & 0 \\
& callus occupying 25\% of defect & 1 \\
& callus occupying 50\% of defect & 2 \\
& callus occupying 75\% of defect & 3 \\
& callus occupying 100\% of defect & 4 \\
Fracture line & Clear & 0 \\
& Relatively clear & 1 \\
& Partial fracture line & 2 \\
& Basically vanished & 3 \\
Bone remodeling & Completely vanished & No bone remodeling \\
\cline { 2 - 3 } & Remodeling of the intramedullary channel & 2 \\
& Full remodeling of cortex & 4 \\
\hline
\end{tabular}

reconstruction of large segmental bone defects of up to $25 \mathrm{~cm}[15,16]$. In the first stage, the defect is temporarily filled with a polymethyl methacrylate (PMMA) spacer. The spacer serves both a mechanical and a biological role. It gives structural support, hinders fibrous invasion, and importantly, induces a vascularized membrane [17]. The induced membrane has been shown to produce growth factors and osteogenic factors, supporting the differentiation and proliferation of bone mesenchymal stem cells (BMSCs) $[18,19]$. Moreover, the induced membrane inhibits soft tissue invasion of the defect, thereby potentially preventing autologous cancellous bone (ACB) resorption [4]. In the second stage, the foreign-body PMMA spacer is removed after 6-8 weeks and the membranous tube is filled with $\mathrm{ACB}$ $[16,20]$. However, ACB is severely restricted by the scarce supply of donors. In addition, regardless of technique, ACB harvesting can also be associated with complications such as bleeding, hematoma, and infection [21-23]. For these disadvantageous reasons, the use of bone substitute would be of great interest, such as $\alpha$-calcium sulfate hemihydrate. 
Table 2. Lane-Sandhu histological score standard

\begin{tabular}{lll}
\hline Category & Standard & Scores \\
\hline Union & No sign of union & 0 \\
& Fibrous union & 1 \\
& Osteochondral union & 2 \\
& Bone union & 3 \\
Spongiosa & Complete reorganization & 4 \\
& No sign of cellular activity & 0 \\
& Early bone formation & 1 \\
& Active new bone formation & 2 \\
Cortex & Reorganized spongiosa formation & 3 \\
& Complete reorganized spongiosa & 4 \\
& Absence of cortex & 0 \\
& Early detection & 1 \\
& Initiation of formation & 2 \\
& Reorganization in majority & 3 \\
\hline
\end{tabular}

In recent years, $\alpha$-calcium sulfate hemihydrate $(\alpha$ $\mathrm{CSH}$ ), an important class of highly cementitious bone substitute, has been clinically used to treat large bone defect. Excellent biocompatibility, biodegradability, and osteoconductivity of $\alpha-\mathrm{CSH}$ biomaterial make it an outstanding bone substitute with properties that are more similar to the autologous bone [24]. Interestingly, it has been found to be potentially osteoinductive like differentiation of BMSCs into osteoblasts [25]. Furthermore, the $\mathrm{pH}$ environment and the ion release behavior of $\alpha-\mathrm{CSH}$ provide an appropriate microenvironment for bone regeneration [26, 27]. However, the resorption rate of $\alpha-\mathrm{CSH}$ is too fast to match the rate of new bone formation.

Based on these studies, we found that the Masquelet technique can produce growth factors and osteogenic factors and protect $\mathrm{ACB}$ against degradation. On the other hand, $\alpha-\mathrm{CSH}$ is a sufficient bone substitute and has excellent biocompatibility, biodegradability, osteoconductivity, and osteoinductivity. In this study, we hypothesized that by replacing or reducing $\mathrm{ACB}$ demanding, the combination of the Masquelet technique and isolated $\alpha-\mathrm{CSH}$ or an $\alpha-\mathrm{CSH} / \mathrm{ACB}$ mix can heal large segmental bone defect, which may represent a promising bone substitute to reduce $\mathrm{ACB}$ demands to treat large segmental bone defect.

\section{Materials and methods}

\section{Animals}

All animal experiments were performed according to protocols approved by the Animal Care and Use Committee of Hainan Medical College and the Use of Laboratory Animals Guideline of the National Institutes of
Health (registration number SYXK 2014-0006). Twentyeight male New Zealand white rabbits (Laboratory Animal Centre of Hainan Medical College, Hainan, China) were used. The rabbits were about $12 \pm 1$ months old and their average weight was $3.5 \pm 0.5 \mathrm{~kg}$.

\section{Experimental design}

The rabbits $(n=28)$ were randomly divided into four experimental groups (each group $n=7$ ): sham group (no implantation), isolated $\alpha$-CSH (Trauma Laboratory, Hainan, China) group, isolated ACB group and $\alpha-\mathrm{CSH} / \mathrm{ACB}$ mix group ( $\alpha-\mathrm{CSH} 50 \%$, ACB 50\%).

\section{Surgical procedure}

The rabbits were given general anesthesia by intravenous injection of $10 \%(w / v)$ chloral hydrate $(3 \mathrm{ml} / \mathrm{kg}$; Solarbio, Beijing, China). Additional regional anesthesia of the brachial plexus with lidocaine hydrochloride was carried out before the osteotomies. The rabbits were positioned in the supine position, and the left limb was prepared with povidone-iodine. A 4-cm longitudinal incision was created along the lower third of the forearm and a 15$\mathrm{mm}$ critical-sized defect was then created in the left distal radius (Fig. 1b). A cylindrical PMMA spacer (Fig. 1a, 4.5- $\mathrm{mm}$ in diameter, $15-\mathrm{mm}$ in length, Simplex P; Kalamazoo, MI, USA) was shaped in vitro and placed into the bone defect (Fig. 1c). The wound was closed with rapidly absorbable $4 / 0$ subcutaneous (Jinhuan, Shanghai, China) and cutaneous sutures and then fixed with a small splint. The second surgical step was performed 6 weeks after the first procedure, under similar anesthetic and experimental conditions. The induced membrane was longitudinally opened and carefully elevated (Fig. 1d). The PMMA spacer was then carefully removed (Fig. 1e). Autogenous cancellous bone was harvested from the iliac crest during the same operation. Then, the cavity delineated by the induced membrane was grafted in line with the experimental design (Fig. 1f). The incised edges of the membrane surrounding the cavity and the muscles were loosely apposed and sutured by simple continuous with rapidly absorbable 4/0 sutures. Cefazolin sodium $(100 \mathrm{mg} / \mathrm{kg})$ was administered intramuscularly at 0,24 , and $48 \mathrm{~h}$ postoperatively. The operated limb was immobilized for 6 weeks using a small splint.

\section{Radiography}

After general anesthesia by intraperitoneal injection of $10 \%(w / v)$ chloral hydrate $(3 \mathrm{ml} / \mathrm{kg})$, anteroposterior and lateral radiographs of the left radius (seven animals per group) were obtained using a portable X-ray machine (40 kV and $6.3 \mathrm{~mA}$; Carestream Health, Inc., Rochester, NY, USA) and digital plates (Agfa; Scoresby VIC, Australia) at 4, 8, and 12 weeks after the second operation. The radiographs were scored using the Lane- 
Mend et al. Journal of Orthopaedic Surgery and Research

(2019) 14:192

Page 4 of 12

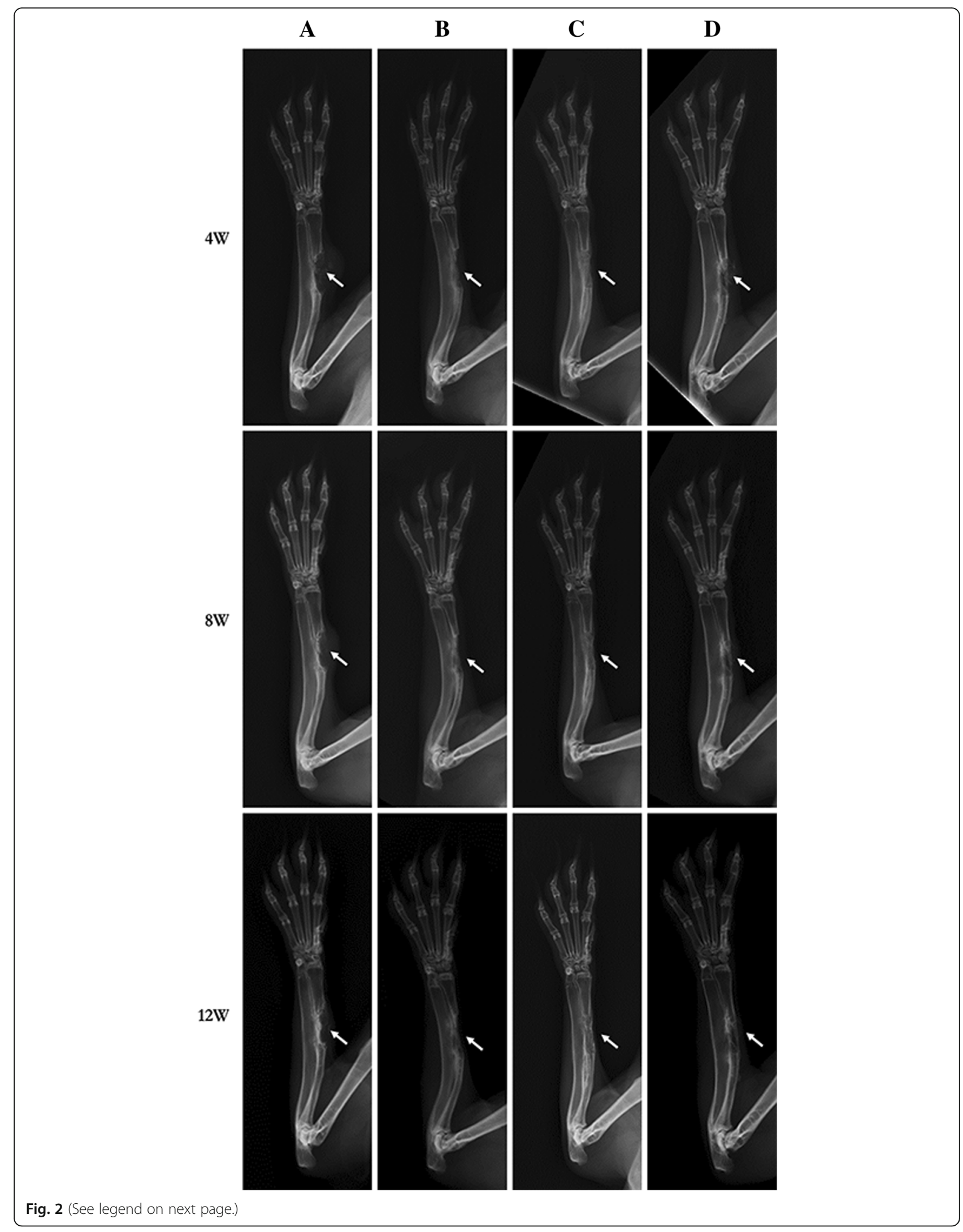


(See figure on previous page.)

Fig. 2 Radiographic assessment of bone formation. The representative radiographs of the bone mineral density in four groups at each time-point. The callus formation and union were observed by direct radiographs taken at 4, 8, and 12 weeks after the second operation. The white arrows over the images indicate the region of interest. a Sham group, $\mathbf{b}$ isolated a-CSH group, $\mathbf{c}$ a-CSH/ACB mix group, $\mathbf{d}$ isolated ACB group, $n=7$

Sandhu scoring system (Table 1). Scoring was carried out by three experts in the department of imagology, who was blind to the status of the rabbits.

\section{Three-dimensional reconstruction of spiral computed tomography}

After general anesthesia by intraperitoneal injection of $10 \%(w / v)$ chloral hydrate $(3 \mathrm{ml} / \mathrm{kg})$, the rabbits (seven animals per group) were positioned in the prone position and scanned using a spiral-computed tomography $(\mathrm{CT})$ multidetector at 12 weeks after the second operation. Using a middle frequency kernel, 1mm-thick axial images underwent three-dimensional (3D) multiplanar reconstruction using the OsiriX MD image processing software (Pixmeo, Geneva, Switzerland) and used to quantify the volume of newly formed bone in the defect. These results were analyzed by a workstation (Philips, Amsterdam, Netherlands) by the same operator blinded to the status of the rabbits.

\section{Histological analysis}

Twelve weeks after the second operation, the rabbits were killed and the samples were collected using a precision saw (Buehler Corporation, Lake Bluff, IL, USA). The samples were fixed in $4 \%$ paraformaldehyde for $48 \mathrm{~h}$, decalcified in 10\% $(w / v)$ EDTA $(\mathrm{pH}$ 7.2-7.4; Solarbio, Shanghai, China) at room temperature for 3 weeks [28], washed with running tap water (purified) overnight, and dehydrated in graded ethanol and embedded in paraffin. Then, the samples were cut into 5 - $\mu$ m-thick microsections in the sagittal plane and stained with Hematoxylin and Eosin (H\&E) staining and Masson's trichrome staining (Solarbio, Shanghai, China), and observed under a light microscope (Nikon, Tokyo, Japan) and photographed (Nikon, Tokyo, Japan). The histological analysis was performed using Lane-Sandhu histological score standard (Table 2). Scoring was carried out by three experts in the department of pathology who was blind to the status of the rabbits.

\section{Alkaline phosphatase assay}

Alkaline phosphatase (ALP) activity was detected by BCIP/NBT alkaline phosphatase color development kit (Beyotime, Shanghai, China) in the bone histological sections. The sections were first deparaffinized in the xylene and rehydrated in graded ethanol and washed with phosphate buffered saline (PBS) three times. A primary polyclonal anti-ALP antibody (Abcam, Cambridge, MA, USA) was used at a 1:200 dilutions and incubated overnight at $4{ }^{\circ} \mathrm{C}$, and then incubated with BCIP/NBT mix detecting reagent for $24 \mathrm{~h}$ at $4{ }^{\circ} \mathrm{C}$. These slides observed under a light microscope and photographed. Semiquantitative analysis of ALP express was performed

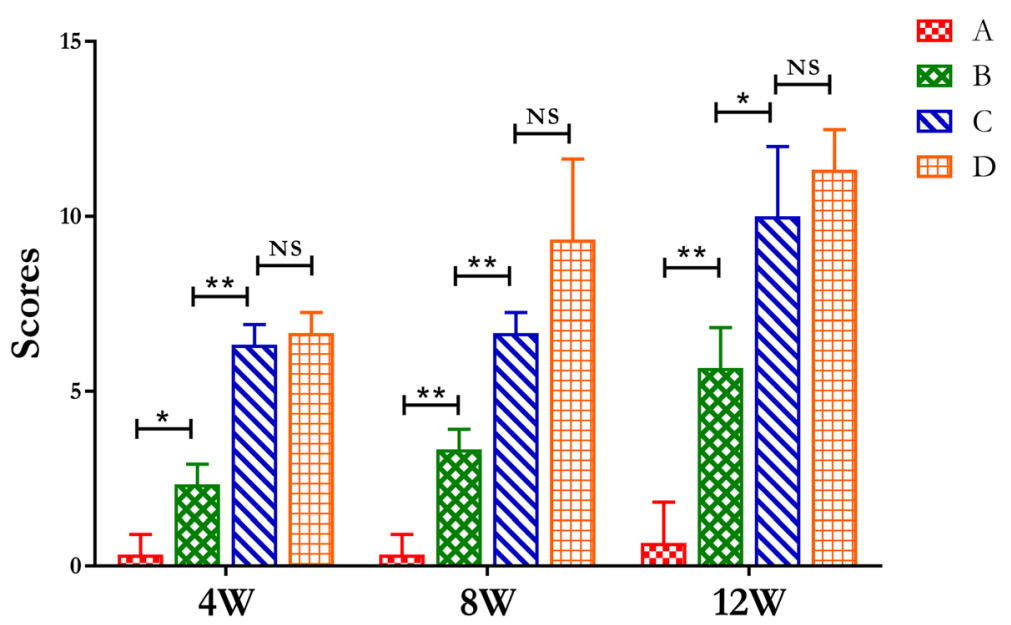

Fig. 3 The radiographic scoring at 4, 8, and 12 weeks after the second operation assessed by the Lane-Sandhu scales. The scoring was obtained by averaging the scores from three different experts. a Sham group, $\mathbf{b}$ isolated a-CSH group, $\mathbf{c}$ a-CSH/ACB mix group, $\mathbf{d}$ isolated ACB group. Data represent mean $\pm \mathrm{SD}$. ${ }^{*} P<0.05 ;{ }^{* *} P<0.01 ; \mathrm{NS}$, not significant; $n=7$ 




Fig. 4 Three-dimensional reconstruction of spiral computed tomography analysis of bone formation at 12 weeks after the second operation. Representative 3D reconstruction of spiral-CT images from sham (a), isolated a-CSH (b), a-CSH/ACB mix (c), and isolated ACB group (d). Quantitative analysis of new bone volume $(\mathbf{e})$. Data represent mean \pm SD. ${ }^{*} P<0.05$; NS not significant; $n=7$

using Image-pro Plus software (Media Cybernetics Inc., Silver Spring, CO, USA)

\section{Immunofluorescence staining}

Immunofluorescence staining was performed to detect the levels of osteocalcin (OCN) expression in the bone histological sections. The sections were first deparaffinized in the xylene and rehydrated in graded ethanol and washed with PBS three times. A primary polyclonal anti-OCN antibody (Abcam, Cambridge, MA, USA) was used at a 1:100 dilutions and incubated overnight at $4{ }^{\circ} \mathrm{C}$, followed by incubation with Alexa Fluor488-conjugated donkey polyclonal secondary antibody (Abcam, Cambridge, MA, USA) at a $1: 1000$ dilutions at $37{ }^{\circ} \mathrm{C}$ for $1 \mathrm{~h}$. The slides were observed by laser scanning confocal microscopy (LSCM, Nikon, Tokyo, Japan). Semiquantitative analysis of OCN express was performed using Image-pro Plus software.

\section{Statistical analysis}

The data were expressed as the means \pm standard deviation (SD). Statistical analysis was performed using oneway ANOVA and Student's $t$ test using SPSS statistical software 19.0 (SPSS Inc., Chicago, IL, USA). Statistical significance was determined at the level of $P<0.05$.

\section{Results}

All surgeries went well, and the rabbits recovered successfully from the operation and remained in good health. The implanted PMMA spacer and $\alpha-\mathrm{CSH}$ did not cause any symptoms of infection or inflammation.

\section{Radiography}

\section{Sham group}

As the results shown (Fig. 2a), bone regeneration in sham group animals was limited to the vicinity of the cut edges of the defects. No bony union was observed radiographically in any animal.

\section{Isolated a-CSH group}

By 12 weeks, complete resorption of the plain $\alpha$-CSH had occurred but this was not accompanied by sufficiently high rates of new bone formation to bridge the gap in any animal (Fig. 2b). Despite some new bone formation, non-union occurred and the bone marrow cavity was not formed in all animals.

\section{a-CSH/ACB mix group}

As early as 4 weeks after implantation, radiographs showed resorption of the $\alpha$-CSH in all animals; this process was complete by the end of 8 weeks. Compared to the isolated $\alpha-\mathrm{CSH}$ group (Fig. $2 \mathrm{~B}$ ), new bone formation occurred in all the $\alpha-\mathrm{CSH} / \mathrm{ACB}$ mix group animals within 12 weeks (Fig. 2c). Sufficient bone had formed within 12 weeks to bridge the gap in all animals. The bone marrow cavity was most re-opened and most remodeling of the cortex was occurred (Fig. 2c).

\section{Isolated $A C B$ group}

The radiopacity of the ACB grafts was initially weaker than the radiopacity of the rest of the radius, but the values increased during subsequent weeks, almost matching that of the host bone by the end of 12 weeks. By that time, the bone union had occurred in all 


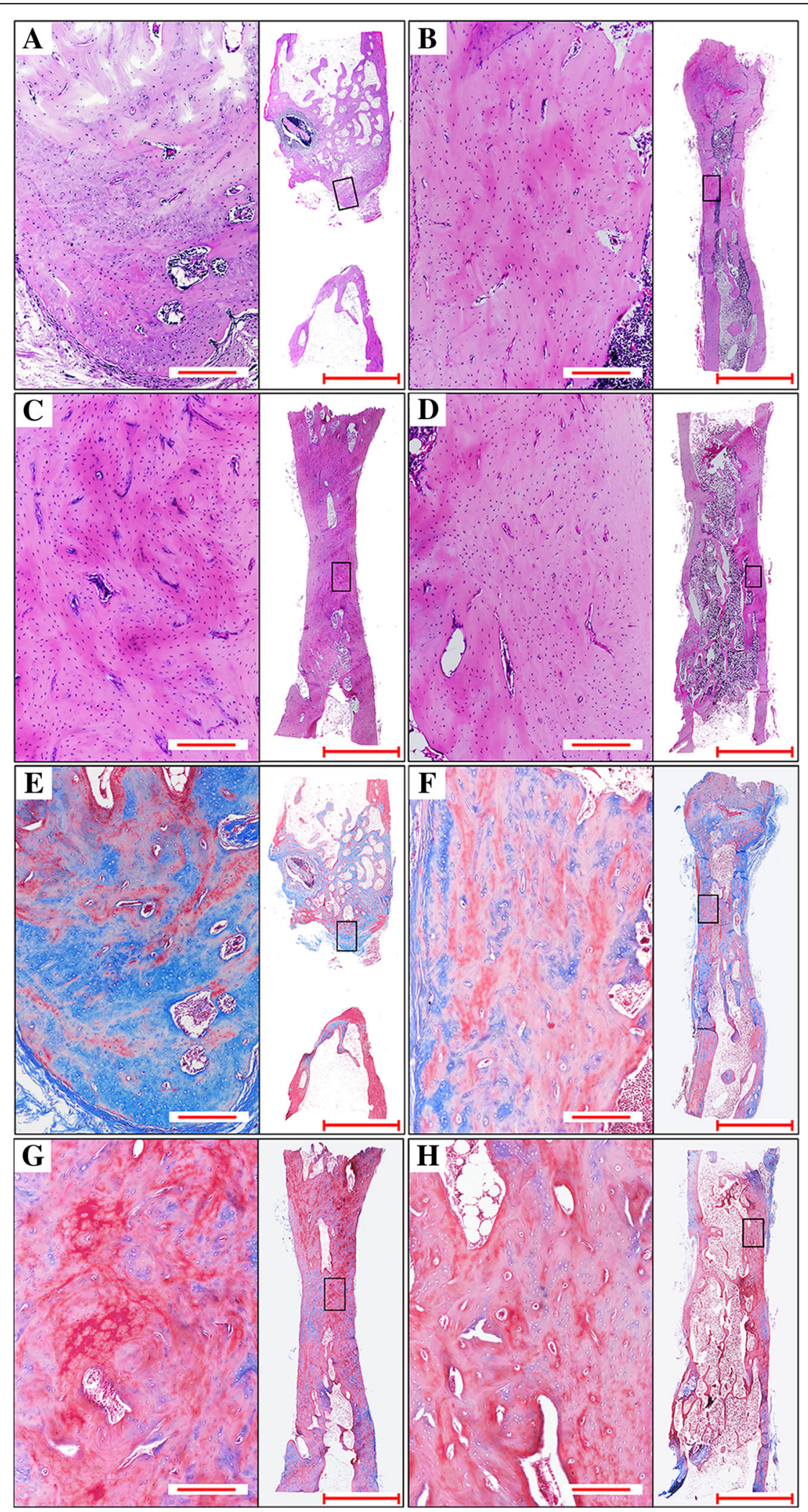

Fig. 5 (See legend on next page.) 
(See figure on previous page.)

Fig. 5 Histological and morphological analysis of bone formation. Representative HE stained (a-d) and Masson stained (e-h) images from sham $(\mathbf{a}, \mathbf{e})$, isolated a-CSH $(\mathrm{B}, \mathrm{F}), \mathrm{a}-\mathrm{CSH} / \mathrm{ABC}$ mix $(\mathbf{c}, \mathbf{g})$ and isolated $\mathrm{ABC}$ group $(\mathbf{d}, \mathbf{h})$. Boxed areas in the HE and Masson stained image at the right side $(\times 40)$ indicate the regions shown in the enlarged HE and Masson stained area at the left side $(\times 100)$. Scale bar $=6000 \mu \mathrm{m}(\times 40)$ and $900 \times \mu \mathrm{m}(\times 100)$

animals. The bone marrow cavity was complete reopened and full remodeling of cortex occurred (Fig. 2d).

Radiological scores of the $\alpha$-CSH/ACB mix group was significantly increased compared to the sham group and the isolated $\alpha-\mathrm{CSH}$ group, while no significant difference compared to the isolated ACB group (Fig. 3).

\section{Spiral-CT}

The 3D structures of the radius were reconstructed, and quantitative analysis was performed on a workstation (Volume Wizard; Philips). As the results shows (Fig. 4), newly formed bone volume in the isolated $\alpha$-CSH group were significantly increased compared to the sham group. Newly formed bone volume in the $\alpha-\mathrm{CSH} / \mathrm{ACB}$ mix group was much higher than the isolated $\alpha$-CSH group. However, there were no significant differences in the newly formed bone volume between the $\alpha$-CSH/ $\mathrm{ACB}$ mix group and the isolated $\mathrm{ACB}$ group.

\section{Histological analysis}

To observe the histological and morphological characteristics, we examined the histological sections by H\&E and Masson's trichrome staining in all groups. As the results shows (Fig. 5), in the sham group, a few new bone was observed in the vicinity of the cut edges of the defects. In the isolated $\alpha-\mathrm{CSH}$ group, the biomaterial was absorbed completely. The previous defects were filled with abundant new bone. The new bone displayed mostly blue collagenous fibers and the bone marrow cavity was not formed. In the $\alpha-\mathrm{CSH} / \mathrm{ACB}$ mix group, the biomaterial was absorbed completely. The volume of new bone is much more than that in the isolated $\alpha$-CSH group and contained more red collagenous fibers than blue ones. The bone marrow cavity was most re-opened. In the isolated $\mathrm{ACB}$ group, the previous defects were filled completely with new bone and red collagenous fibers much more than the $\alpha-\mathrm{CSH} / \mathrm{ACB}$ mix group, which indicated that the new bone were undergoing gradual calcification and maturation. The bone marrow cavity was completely re-opened. Histological scores of the $\alpha$-CSH/ACB mix group was significantly increased compared to the sham group and the isolated $\alpha$-CSH group, while no significant difference compared to the isolated ACB group (Fig. 6).

\section{Alkaline phosphatase assay}

To determine the osteogenic activity of osteoblast, we examined the alkaline phosphatase (ALP) expression levels in all groups. As the results shown (Fig. 7), the levels of ALP expression were more prevalently expressed within the defects in the isolated $\alpha-\mathrm{CSH}, \alpha-$ $\mathrm{CSH} / \mathrm{ACB}$ mix, and isolated $\mathrm{ACB}$ group compared to the sham group, and higher expression of ALP was observed in the $\alpha-\mathrm{CSH} / \mathrm{ACB}$ mix group and the isolated $\mathrm{ACB}$ group. However, there were no significant

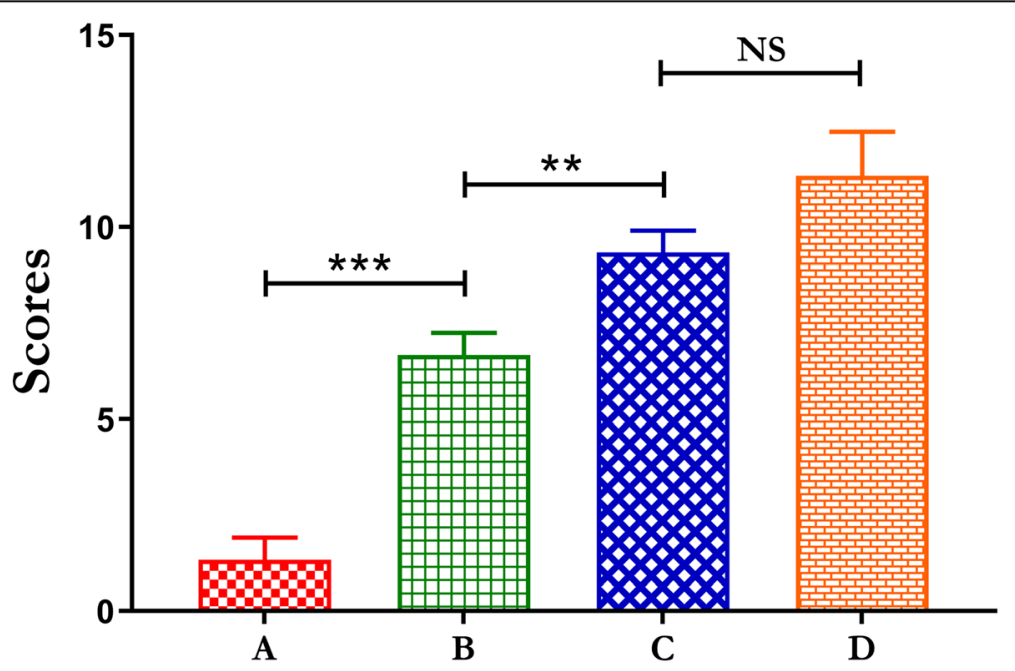

Fig. 6 The histological scoring 12 weeks after the second operation assessed by the Lane-Sandhu histological score standard. The scoring was obtained by averaging the scores from three different experts. a Sham group, $\mathbf{b}$ isolated $\mathbf{a}-\mathrm{CSH}$ group, $\mathbf{c}$ a-CSH/ACB mix group, $\mathbf{d}$ isolated ACB group. Data represent mean $\pm S D$. ${ }^{* *} P<0.01 ;{ }^{* *} P<0.001$; NS not significant; $n=7$ 


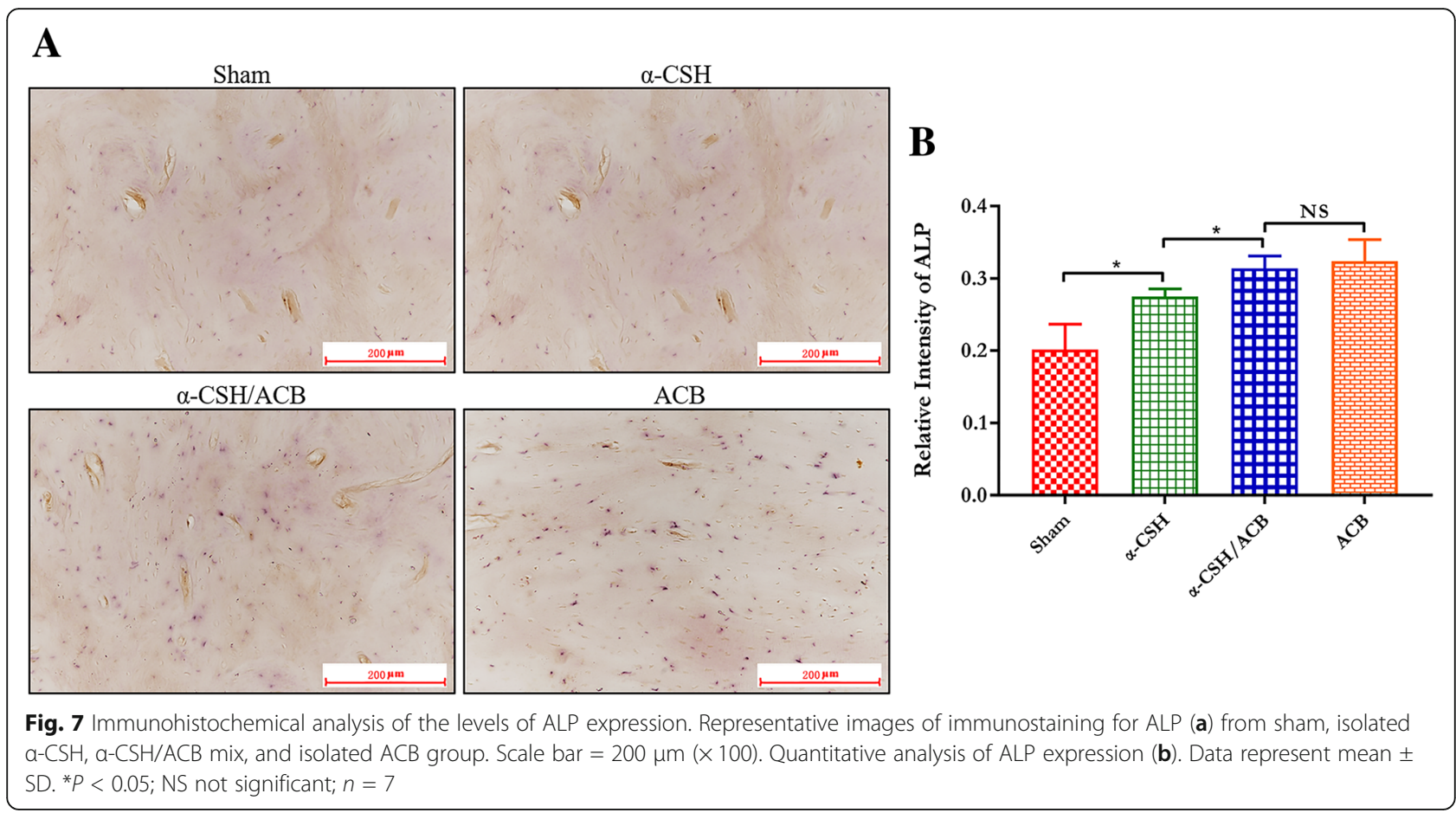

differences in the level of ALP between the $\alpha-\mathrm{CSH} / \mathrm{ACB}$ mix group and the isolated $\mathrm{ACB}$ group.

\section{Immunofluorescence staining}

To further determine the newly formed bone tissue, we examined the level of OCN expression by immunofluorescence staining. As the results shows (Fig. 8), the protein expression of $\mathrm{OCN}$ was significantly increased in three other groups compared to the sham group, and higher expression of OCN was observed in the $\alpha-\mathrm{CSH} /$ $\mathrm{ACB}$ mix and isolated $\mathrm{ACB}$ group. However, there were no significant differences in the level of $\mathrm{OCN}$ between the $\alpha-\mathrm{CSH} / \mathrm{ACB}$ mix group and the isolated ACB group.

\section{Discussion}

Reconstruction of large segmental bone defects remains a difficult challenge for orthopedic and trauma surgeons. Current solutions to manage large segmental bone defects include vascularized bone transfer and the Ilizarov intercalary bone method. However, these approaches have limitations because they are microsurgically and technically demanding. The Masquelet technique with ACB graft is a novel approach allowing reconstruction of large segmental bone defects $[15,29,30]$. Several research have highlighted the advantages of using the Masquelet technique: (1) maintain of $\mathrm{ACB}$ volume over time through protection of the $\mathrm{ACB}$ against resorption [4]; (2) restraint of the ACB in place [31]; (3) inhibit soft tissue invasion into the defect [18]; (4) produce growth factors and osteogenic factors, supporting the proliferation and differentiation of BMSCs [19, 32]. However, the Masquelet technique has limitations because of the supply of $\mathrm{ACB}$ and the complications of donor site [21-23]. Therefore, the use of bone substitute would be of great interest.

To the best of our knowledge, which is the most appropriate bone substitute remains controversial. Bone substitute must be selected for their properties to optimize bone repair, promote cell survival, proliferation, and differentiation, while exhibiting appropriate degradation rate, mechanical strength, excellent osteoconductivity, and osteoinductivity [33]. $\alpha-\mathrm{CSH}$ is an outstanding bone substitute due to its easy availability, good biocompatibility, biodegradability, osteoconductivity, and a long history of use in bone reconstruction [34-36]. Moreover, it has been found to be potentially osteoinductive-like differentiation of BMSCs into osteoblasts [25]. A probable mechanism by which $\alpha-\mathrm{CSH}$ can promote bone regeneration is by maintaining a high concentration of extracellular calcium ions, which is well known to have the effects of promoting osteoblast activity and inhibiting osteoclast activity [37-39]. However, the resorption rate of $\alpha-\mathrm{CSH}$ is too fast to match the rate of new bone formation.

In this study, the combination of the Masquelet technique and isolated $\alpha-\mathrm{CSH}$ or an $\alpha-\mathrm{CSH} / \mathrm{ACB}$ mix was investigated in a rabbit critical-sized defect model as they possessed the combined effects-sufficient supply of $\alpha-\mathrm{CSH}$ and low resorption of the Masquelet technique. A practical definition of a critical-sized bone 


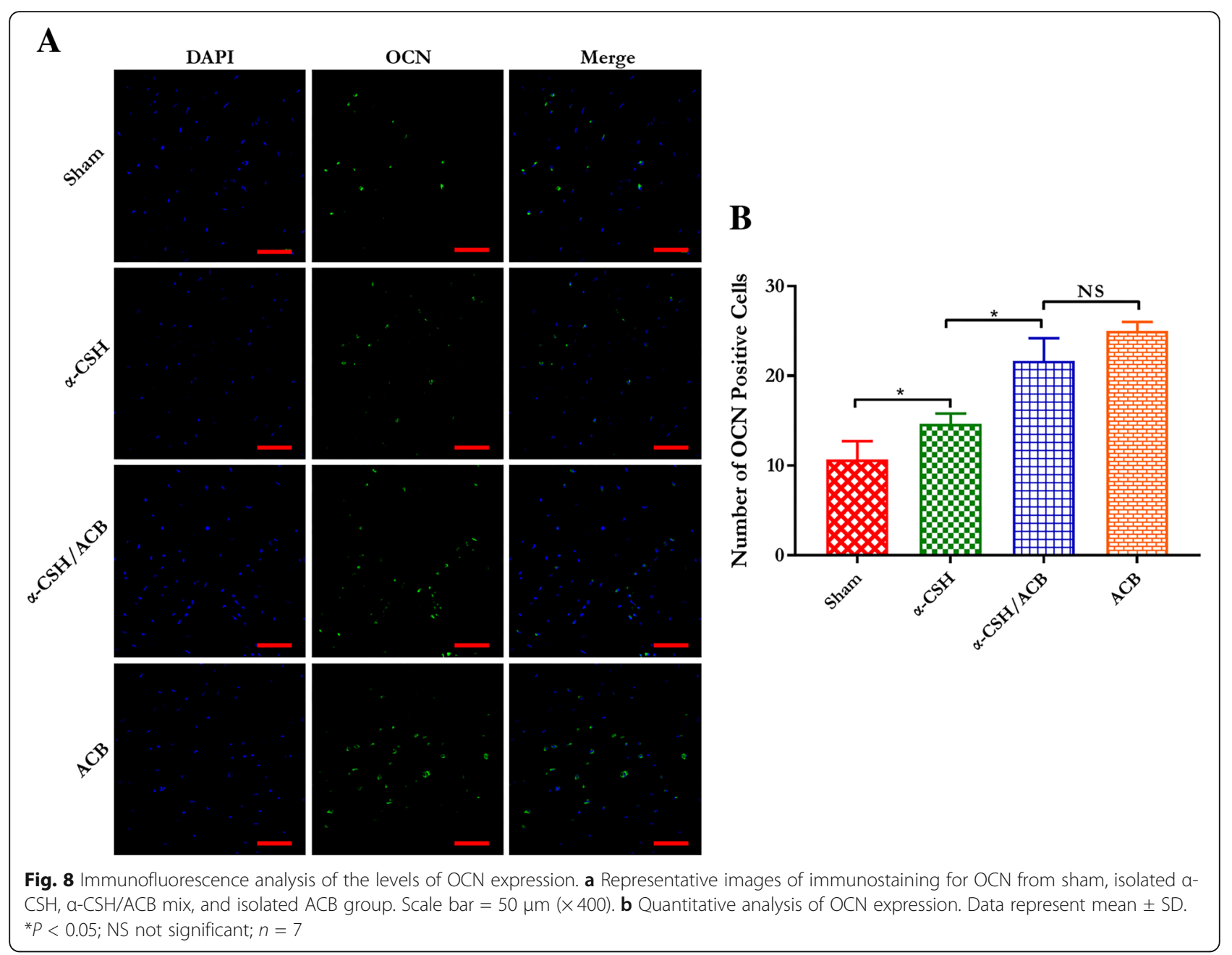

defect was suggested as a segmental bone defect of a length exceeding 2 to 2.5 times the diameter of the affected bone and will not heal spontaneously during the lifetime $[17,40]$. To evaluate the optimal alternative solutions, we designed four groups: sham, isolated $\alpha-\mathrm{CSH}$, $\alpha-\mathrm{CSH} / \mathrm{ACB} \operatorname{mix}(1: 1, w / w)$, and isolated ACB group. The sham group is the negative control group to confirm that the critical-sized defect cannot heal spontaneously. The isolated ACB group is the positive control group to confirm that the critical-sized defect can heal using isolated ACB grafts. Our results showed that the sham group did not heal spontaneously. The Masquelet technique in conjunction with isolated $\alpha-\mathrm{CSH}$ can partially heal critical-sized bone defect, while $\alpha$-CSH/ACB mix $(1: 1, w / w)$ can approximately complete heal criticalsized bone defect. These results demonstrated that the Masquelet technique in conjunction with isolated $\alpha$ $\mathrm{CSH}$ does not have enough capacity to repair a criticalsized defect. The bone repair capacity of $\alpha-\mathrm{CSH} / \mathrm{ACB}$ $\operatorname{mix}(1: 1, w / w)$ is similar to isolated ACB $(P>0.05)$. This suggests that $\alpha-\mathrm{CSH}$ can replace at least half of $\mathrm{ACB}$, thus reducing the supply of $\mathrm{ACB}$ and the complication of donor site. In a further study, more ratio of $\alpha-\mathrm{CSH} /$ ACB mixture should be investigated. In addition, $\alpha-\mathrm{CSH}$ or $\alpha-\mathrm{CSH} / \mathrm{ACB}$ mix can be doped with osteogenic factors like bone morphogenetic protein (BMP) and vascular endothelial growth factor (VEGF) to further improve its ability to promote bone repair.

To further investigate the capacity of new bone formation of $\alpha-\mathrm{CSH}$ at a molecular level, we examined the expression levels of ALP and OCN. Osteoblasts play a key role in bone formation [41]. ALP and OCN, known as the most abundant bone matrix enzyme and protein, are preferentially expressed by osteoblasts [42]. Therefore, ALP and OCN are often used as a typical biomarker for bone formation and maturation [43]. Our results showed that the ALP and OCN expression of the $\alpha-\mathrm{CSH} / \mathrm{ACB}$ mix group were higher than the isolated $\alpha-\mathrm{CSH}$ group $(P<0.05)$ and were similar to the isolated ACB group $(P$ $s>0.05)$. These results suggest that $\alpha-\mathrm{CSH}$ and $\alpha-\mathrm{CSH} /$ ACB mix can stimulate ALP and OCN expressions and then improve bone formation. In a further study, the 
definitive mechanism for stimulating ALP and OCN expression should be investigated.

In the first stage of the Masquelet technique, a PMMA spacer is inserted into the bone defect during surgery. In clinical practices, the spacer is molded in vivo. In this study, the spacer is molded in vitro. There is a shortcoming that the spacer does not completely match the diameter and length of the bone defect, which may affect the stabilization of the spacer and the formation of the induced membrane. In addition, another limitation of this study was the lack of an external fixation or a plate, as rabbit radius is too small to match the size of human external fixation and plate. Instead, a small splint fixation was used to enhance the stabilization of the PMMA spacer at the bone defect.

In the second stage of the Masquelet technique, we used $\alpha$-CSH granules to fill the membranous tubes. In clinical practice, the shape and size of patients' defects are highly variable. Standardized granules could fill defects of any size and shape and thus abolish the complex computer-assisted customization [44]. In a further study, which is the optimal size and shape of $\alpha-\mathrm{CSH}$ or other bone substitute should be investigated.

\section{Conclusion}

In conclusion, this study demonstrates that the Masquelet technique in conjunction with $\alpha$-CSH/ACB mix (1:1, $w / w)$ can complete repair rabbit critical-sized bone defect. When the amount of ACB is not sufficient in clinical practice, $\alpha-\mathrm{CSH}$ might be a promising bone substitute to replace at least half of ACB to repair large segmental bone defect.

\section{Abbreviations}

ACB: Autologous cancellous bone; ALP: Alkaline phosphatase; BMSCs: Bone mesenchymal stem cells; OCN: Osteocalcin; PBS: Phosphate buffered saline; PMMA: Polymethyl methacrylate; a-CSH: a-Calcium sulfate hemihydrate

\section{Acknowledgements}

Not applicable.

\section{Authors' contributions}

ZLM and LP drafted the first manuscript. ZLM, ZQW, BXS, HBL, YYB, DLZ, JF, and LP participated in the study design, data acquisition, analysis and interpretation of data, critical review, and final approval. All authors read and approved the final manuscript.

\section{Funding}

This work was supported by National Natural Science Foundation of China (nos. 81460339 and 81760291), University Student Innovation and Entrepreneurship Project (201711810002), First Affiliated Hospital Cultivation Fund of Hainan Medical University (HYFYPY201805) Scientific Research Project of Hainan Provincial Health and Family Planning Commission (18A200151), and Key Scientific Research Projects of Hainan Higher Education Institutions (Hnky2019ZD-28).

\section{Availability of data and materials}

The datasets used and/or analyzed during the current study are available from the corresponding author on reasonable request.
Ethics approval and consent to participate

This study was approved by the Animal Care and Use Committee of Hainan Medical College.

\section{Consent for publication \\ Not applicable.}

\section{Competing interests}

The authors declare that they have no competing interests.

\section{Author details}

${ }^{1}$ Municipal Hospital Affiliated to Medical School of Taizhou University, Taizhou, China. ${ }^{2}$ Department of Trauma Center, The First Affiliated Hospital of Hainan Medical University, Haikou, China. ${ }^{3}$ School of Pharmaceutical Sciences, Wenzhou Medical University, Wenzhou, China.

Received: 17 October 2018 Accepted: 11 June 2019

Published online: 26 June 2019

\section{References}

1. Pobloth AM, Schell H, Petersen A, Beierlein K, Kleber C, Schmidt-Bleek K, Duda GN. Tubular open-porous beta-tricalcium phosphate polycaprolactone scaffolds as guiding structure for segmental bone defect regeneration in a novel sheep model. J Tissue Eng Regen Med. 2018;12(4):897-911.

2. Lasanianos NG, Kanakaris NK, Giannoudis PV. Current management of long bone large segmental defects. Orthopaedics Trauma. 2010;24(2):149-63.

3. Minier K, Touré A, Fusellier M, Fellah B, Bouvy B, Weiss P, Gauthier O. BMP-2 delivered from a self-crosslinkable $\mathrm{CaP} /$ hydrogel construct promotes bone regeneration in a critical-size segmental defect model of non-union in dogs. Vet Comp Orthop Traumatol. 2014:27(6):411-21.

4. Henrich D, Seebach C, Nau C, Basan S, Relja B, Wilhelm K, Schaible A, Frank J, Barker J, Marzi I. Establishment and characterization of the Masquelet induced membrane technique in a rat femur critical-sized defect model. J Tissue Eng Regen Med. 2016;10(10):382-96.

5. Mauffrey C, Barlow BT, Smith W. Management of segmental bone defects. J Am Acad Orthopaedic Surg. 2015:23(3):143-53.

6. Friedrich JB, Moran SL, Bishop AT, Shin AY. Free vascularized fibula grafts for salvage of failed oncologic long bone reconstruction and pathologic fractures. Microsurgery. 2009;29(5):385-92.

7. Pederson WC, Person DW. Long bone reconstruction with vascularized bone grafts. Orthopedic Clin North Am. 2007;38(1):23-35.

8. Ghert M, Colterjohn N, Manfrini M. The use of free vascularized fibular grafts in skeletal reconstruction for bone tumors in children. J Am Acad Orthopaedic Surg. 2007;15(10):577-87.

9. Paley D, Maar DC. Ilizarov bone transport treatment for tibial defects. J Orthopaedic Trauma. 2000;14(2):76.

10. Aronson J. Limb-lengthening, skeletal reconstruction, and bone transport with the llizarov method. J Bone Joint Surg Am. 1997;79(8):1243.

11. Mercado-Pagán ÁE, Stahl AM, Shanjani Y, Yang Y. Vascularization in bone tissue engineering constructs. Ann Biomed Eng. 2015;43(3):718-29.

12. Mekhail AO, Abraham E, Gruber B, Gonzalez M. Bone transport in the management of posttraumatic bone defects in the lower extremity. J Traum. 2004;56(2):368

13. Pelissier P, Martin D, Baudet J, Lepreux S, Masquelet AC. Behaviour of cancellous bone graft placed in induced membranes. Br J Plast Surg. 2002 55(7):596-8.

14. Masquelet AC, Fitoussi F, Begue T, Muller GP. Reconstruction of the long bones by the induced membrane and spongy autograft. Ann Chir Plast Esthet. 2000;45(3):346-53.

15. Gouron R, Petit L, Boudot C, Six I, Brazier M, Kamel S, Mentaverri R. Osteoclasts and their precursors are present in the induced-membrane during bone reconstruction using the Masquelet technique. J Tissue Eng Regen Med. 2017;11(2):382.

16. Masquelet AC, Begue T. The Concept of Induced Membrane for Reconstruction of Long Bone Defects. Orthop Clin North Am. 2010; 41(1):27-37

17. Bosemark $\mathrm{P}$, Perdikouri $\mathrm{C}$, Pelkonen $\mathrm{M}$, Isaksson $\mathrm{H}$. The Masquelet induced membrane technique with BMP anda synthetic scaffold can heal a rat femoral critical size defect. J Orthopaed Res Off Publ Orthopaed Res Soc. 2015;33(4):488-95. 
18. Taylor BC, French BG, Fowler T,, Russell J, Poka A. Induced membrane technique for reconstruction to manage bone loss. J Am Acad Orthop Surg. 2012;20(3):142-50

19. Pelissier P, Masquelet AC, Bareille R, Pelissier SM, Amedee J. Induced membranes secrete growth factors including vascular and osteoinductive factors and could stimulate bone regeneration. J Orthop Res. 2004;22(1):73-9.

20. Mauffrey C, Hake ME, Chadayammuri V, Masquelet AC. Reconstruction of long bone infections using the induced membrane technique: tips and tricks. J Orthop Trauma. 2016;30(6):188-93.

21. Dimitriou R, Mataliotakis Gl, Angoules AG, Kanakaris NK, Giannoudis PV. Complications following autologous bone graft harvesting from the iliac crest and using the RIA: a systematic review. Injury. 2011;42(Suppl 2):3-15.

22. Younger EM, Chapman MW. Morbidity at bone graft donor sites. J Orthop Trauma. 1989;3(3):192-5.

23. Arrington ED, Smith WJ, Chambers HG, Bucknell AL, Davino NA. Complications of iliac crest bone graft harvesting. Clin Orthopaed Relat Res. 1996:329(329):300-9.

24. Li X, Xu CP, Hou YL, Song JQ, Cui Z, Wang SN, Huang L, Zhou CR, Yu B. A novel resorbable strontium-containing alpha-calcium sulfate hemihydrate bone substitute: a preparation and preliminary study. Biomed Mater. 2014; 9(4):45010.

25. Young-Kyun K, Ji-Young L, Su-Gwan K, Seung-Chul L. Guided bone regeneration using demineralized allogenic bone matrix with calcium sulfate: case series. J Adv Prosthodont. 2013;5(2):167.

26. Stubbs D, Deakin M, Chapman-Sheath P, Bruce W, Debes J, Gillies RM, Walsh WR. In vivo evaluation of resorbable bone graft substitutes in a rabbit tibial defect model. Biomaterials. 2004;25(20):5037-44.

27. Orsini G, Ricci J, Scarano A, Pecora G, Petrone G, lezzi G, Piattelli A. Bonedefect healing with calcium-sulfate particles and cement: an experimental study in rabbit. J Biomed Mater Res B Appl Biomater. 2004;68B(2):199-208.

28. Jing Z, Wu Y, Su W, Tian M, Jiang W, Cao L, Zhao L, Zhao Z. Carbon nanotube reinforced collagen/hydroxyapatite scaffolds improve bone tissue formation In Vitro and In Vivo. Ann Biomed Eng. 2017:45(9):2075-87.

29. Woon CY, Chong KW, Wong MK. Induced membranes - a staged technique of bone-grafting for segmental bone loss: a report of two cases and a literature review. J Bone Joint Surg Am. 2010;92(1):196.

30. Apard T, Bigorre N, Cronier P, Duteille F, Bizot P, Massin P. Two-stage reconstruction of post-traumatic segmental tibia bone loss with nailing. Orthop Traumatol Surg Res. 2010;96(5):549.

31. Masquelet AC. Muscle reconstruction in reconstructive surgery: soft tissue repair and long bone reconstruction. Langenbecks Arch Surg. 2003;388(5): 344-6.

32. Viateau V, Guillemin G, Yang YC, Bensaid W, Reviron T, Oudina K, Meunier A, Sedel L, Petite H. A technique for creating critical-size defects in the metatarsus of sheep for use in investigation of healing of long-bone defects. Am J Vet Res. 2004;65(12):1653-7.

33. Frasca S, Norol F, Visage CL, Collombet JM, Letourneur D, Holy X, Ali ES. Calcium-phosphate ceramics and polysaccharide-based hydrogel scaffolds combined with mesenchymal stem cell differently support bone repair in rats. J Mater Sci Mater Med. 2017;28(2):35.

34. Walsh WR, Morberg P, Yu Y, Yang JL, Haggard W, Sheath PC, Svehla M, Bruce WJ. Response of a calcium sulfate bone graft substitute in a confined cancellous defect. Clin Orthop Relat Res. 2003:406(406):228.

35. Beuerlein MJ, Mckee MD. Calcium sulfates: what is the evidence? J Orthop Trauma. 2010;24(Suppl 1):46-51.

36. Shen Y, Yang S, Liu J, Xu H, Shi Z, Lin Z, Ying X, Guo P, Lin T, Yan S. Engineering scaffolds integrated with calcium sulfate and oyster shell for enhanced bone tissue regeneration. ACS Appl Mater Interfaces. 2014;6(15):12177.

37. Kanatani M, Sugimoto T, Kanzawa M, Yano S, Chihara K. High extracellular calcium inhibits osteoclast-like cell formation by directly acting on the calcium-sensing receptor existing in osteoclast precursor cells. Biochem Biophys Res Commun. 1999;261(1):144-8.

38. Nakade O, Takahashi K, Takuma T, Aoki T, Kaku T. Effect of extracellular calcium on the gene expression of bone morphogenetic protein-2 and -4 of normal human bone cells. J Bone Miner Metab. 2001;19(1):13-9.

39. Thomas MV, Puleo DA, Alsabbagh M. Calcium sulfate: a review. J Long Term Eff Med Impl. 2005;15(6):599-607.

40. Wiese A, Pape HC. Bone defects caused by high-energy injuries, bone loss, infected nonunions, and nonunions. Orthop Clin North Am. 2010;41(1):1.

41. Ho MH, Yao CJ, Liao MH, Lin PI, Liu SH, Chen RM. Chitosan nanofiber scaffold improves bone healing via stimulating trabecular bone production due to upregulation of the Runx2/osteocalcin/alkaline phosphatase signaling pathway. Int J Nanomedicine. 2015;10:5941-54.

42. Wolf $\mathrm{G}$. Function of the bone protein osteocalcin: definitive evidence. Nutr Rev. 1996:54(10):332-3.

43. Boskey AL, Wians F Jr, Hauschka PV. The effect of osteocalcin on in vitro lipid-induced hydroxyapatite formation and seeded hydroxyapatite growth. Calcif Tissue Int. 1985;37(1):57-62.

44. Viateau V, Guillemin G, Bousson V, Oudina K, Hannouche D, Sedel L, Logeart-Avramoglou D, Petite H. Long-bone critical-size defects treated with tissue-engineered grafts: a study on sheep. J Orthop Res. 2007;25(6):741-9.

\section{Publisher's Note}

Springer Nature remains neutral with regard to jurisdictional claims in published maps and institutional affiliations.

\section{Ready to submit your research? Choose BMC and benefit from:}

- fast, convenient online submission

- thorough peer review by experienced researchers in your field

- rapid publication on acceptance

- support for research data, including large and complex data types

- gold Open Access which fosters wider collaboration and increased citations

- maximum visibility for your research: over $100 \mathrm{M}$ website views per year

At BMC, research is always in progress.

Learn more biomedcentral.com/submissions 